This is an author produced version of a paper published in Canadian Journal of Forest Research.

This paper has been peer-reviewed and is proof-corrected, but does not include the journal pagination.

Citation for the published paper:

Karin Öhman, Lars Edenius \& Grzegorz Mikusiński. (2011) Optimizing spatial habitat suitability and timber revenue in long-term forest planning. Canadian Journal of Forest Research. Volume: 41, Number: 3, pp 543-551.

http://dx.doi.org/10.1139/X10-232.

Access to the published version may require journal subscription. Published with permission from: NRC Research Press.

Standard set statement from the publisher:

This is an author's version of an article published in Canadian Journal of Forest Research, 7 februari 2011. The article is available online at: http://www.nrcresearchpress.com/doi/abs/10.1139/x10-232\#.UMcek4N97fs

Epsilon Open Archive http://epsilon.slu.se 


\section{Optimizing spatial habitat suitability and timber revenue in long-term forest planning}

Karin Öhman $^{\mathrm{a}^{*}}$, Lars Edenius ${ }^{\mathrm{b}}$ and Grzegorz Mikusiński ${ }^{\mathrm{c}, \mathrm{d}}$.

a) Department of Forest Resource Management

SLU, SE- 90183 Umeå

Sweden

Email: Karin.ohman@slu.se

telephone +46 (0) 90-786 85 88, fax +4690778116

b) Department of Wildlife, Fish, and Environmental Studies, Swedish University of Agricultural, SE-901 83 Umeå

Email: lars.edenius@slu.se,

telephone +46 (0) 90- 7868341

c) Grimsö Wildlife Research Station, Department of Ecology, Swedish University of Agricultural, SE-7310 91 Riddarhyttan, Sweden

Email: grzegorz.mikusinski@slu.se

telephone +46 (0) 581-697329

d) School for Forest Management, Swedish University of Agricultural, Box 43,

SE-739 21 Skinnskatteberg, Sweden

*corresponding author 


\section{Abstract:}

Effective tools must be developed that include consideration of biodiversity in the traditional forest planning process. The objective of this study is to present a spatial habitat suitability model that could be included in the optimization of longterm forest planning where the problem can be solved with an exact solution method. This could be an advantage, since, e.g. many forest planning systems available today are designed for problems that could be solved with an exact solution method. The habitat model consists of two parts: suitability assessment of stand-wise conditions and spatial conditions. To investigate whether the model works in a realistic setting, we used a case study and applied the model to the habitat demands for Hazel Grouse (Bonasa bonasia L.). The results from the case study indicate that the model is effective for including spatial habitat consideration and that the model could be used for creating different degrees of the clustering of habitats. Further, the loss in net present value as a result of the spatial habitat demands was limited in the case study. We suggest that this modeling approach could be extended to other species with large area requirements and add to the existing tools for forest biodiversity assessment in forest management planning.

Keywords: Hazel Grouse, mixed-integer programming, optimization, spatial planning 


\section{Introduction}

Since many forest species and habitats are considered to be under threat from forestry aiming at timber production, biodiversity issues have become important focal points in strategic and tactical planning for sustainable forest management (Heywood, 1995; Fries et al., 1998; Larsson and Danell, 2001; Tittler et al., 2001). One tool for including consideration to biodiversity in the traditional forest planning process is habitat suitability models (Marzluff et al., 2002; Larson et al., 2004; Edenius and Mikusiński 2006; Spies et al., 2007). Habitat suitability denotes the capability of an area to provide conditions for species to meet their needs for food, shelter, and reproduction (Turner et al., 2001). It could be envisioned as an "envelope of environmental and vegetation requirements within the species" (O’Connor, 2002). Because inclusive habitat descriptors may be extracted from variables stored in forest databases (e.g. age of forest and tree species composition), habitat suitability modeling has appeal for forest management planning (Felix et al., 2004; Edenius and Mikusiński, 2006). In the case of species with limited area demands, simple tabulation of forest characteristics may suffice to quantify habitat suitability. However, for species with area requirements that exceed the size of a typical forest stand, habitat planning must be performed at the landscape scale, i.e. encompass several forest stands simultaneously (e.g. Larson et al., 2004). Such landscape simulations are capable of detecting temporal bottlenecks in future availability of habitat for model species and thereby avoid management situations that provide inadequate habitat for the species in a given landscape (Mikusiński and Edenius, 2006). 
The focus when using habitat suitability models (hereafter habitat models) in forest planning is primarily to investigate the potential habitat area for certain species if a certain management plan is applied (i.e. an answer for the "what if" question)(e.g. Gustafson et al., 2006; Vospernik and Reimoser, 2008). Another approach is to include the habitat models in the problem formulation and the optimization (i.e. an answer for the "how to" question). How should the forest area be managed if a certain area of habitat is desired? This question assumes that the habitat demand is included in the optimization part of the planning process. Unfortunately, when habitat models are included in the optimization, the complexity of the management problem will increase, depending on if the habitat model requires spatial information or not. In general, the first step in the optimization process is to simulate yields for different treatment schedules ${ }^{1}$ for a number of values, e.g. standing volume for different species and harvest volume. The next step is to select a schedule for each stand with the help of an optimization method so that the objective is maximized or minimized with consideration to the constraints. With this approach, the time-consuming simulation does not need to be done for each combination of treatment schedules. However, this approach requires either optimization methods that could consider spatial relationships between stands (e.g. for species with large area requirements) or the use of non-spatial habitat models. In a non spatial habitat model, only information about the stand is required (e.g. stand age, tree species composition) and no information about the neighborhood is needed to decide whether a stand consists of suitable habitat or not (e.g. for species without area requirements). The definition of whether a stand consists of habitat for a certain management activity

\footnotetext{
${ }^{1} \mathrm{~A}$ treatment schedule is a sequence of treatments e.g. regeneration, thinning, clear cutting or doing nothing, for a planning unit from period 1 to the end of the planning horizon.
} 
in a period could be decided during the simulation phase. In such cases, the amount of habitat could be expressed as a linear sum of the area over the treatment schedules for the landscape and the resulting planning problem could be solved with linear programming, which is a traditional optimization method for solving linear problems. On the other hand, in spatial habitat models, information about the conditions in the stand and the conditions in surrounding areas is needed for deciding whether the stand qualifies as a habitat. The complexity of the management problem will then increase, since whether a stand qualifies as a habitat or not will depend not only on the management activities in the stand but also on the activities in surrounding stands. The amount of habitat can no longer be expressed as a linear combination of the treatment schedules over the whole landscape. As a result, when including spatial habitat demands in the optimization, traditional linear programming with continuous variables cannot be used.

Many studies that included spatial habitat models in the optimization have solved the stated management problem with a heuristic method (e.g. Bettinger et al., 2003; Hurme et al., 2007; Tikkanen et al., 2007). The reason for this, is e.g. that integer variables must be used, the non linear relationships or the limited size of the problems that can be solved with an exact solution method. However, even if heuristic methods are designed for solving complex problems and nonlinear relationships, a heuristic method cannot guarantee that the optimal solution to a specified problem will be found, and must be specially designed for, or at least adapted to, the specific problem. An alternative to heuristic methods is to use an exact solution method, such as mixed-integer programming with the branch and bound algorithm (e.g. Hof et al., 1994; Rebain and McDill, 2003; Wei and 
Hoganson, 2007; Öhman and Wikström, 2008; Zhang et al. 2009). This approach has a number of advantages. First, the optimal solution to the specified problem can be found. Second, the parameterization connected to using a heuristic method, which often is time-consuming and sensitive both to problem type and forest conditions, is avoided. Further, many forest planning systems available today are designed for problems that could be solved with an exact solution method (Heureka, 2008).Traditionally, one limitation when using exact solution methods has been the time required for solving complex problems and, connected to this, the limited size of the problems that can be solved. However, recent developments in optimization software systems and computer hardware have increased the scope for solving large scale problems within a reasonable time (Johnson et al. 2000; Atamtürk and Svavelsbergh 2005).

The objective of this study is to present a spatial habitat model that could be included in the optimization of long-term forest planning where the problem can be solved with an exact solution method. The habitat model used in this study includes both suitability assessment of stand-wise conditions and evaluation of spatial context of the stand in a broader landscape. The model gives the decision maker possibilities to test a range of plans with different spatial layouts (i.e. different degrees of spatial aggregation of habitat). To investigate whether the model works in a realistic setting, we used a case study from northern Sweden and applied the model to the habitat demands of Hazel Grouse (Bonasa bonasia L.), a relatively specialized forest grouse species with respect to habitat requirements. In the case study the resulting management problem is solved with a traditional branch and bound algorithm, which is a standard algorithm for solving mixedinteger problems. 


\section{Materials and Methods}

\section{Model formulation}

The habitat model for describing if a particular stand is a habitat consists of two parts: stand-wise conditions and spatial conditions. Both types of conditions must be fulfilled before a stand qualifies as a habitat. The stand-wise conditions are dependent only on the selected management for the stand. Depending on the needs of the species, a number of conditions should be fulfilled (e.g. the stand should have a certain age, volume, tree species composition, etc.) before the stand could qualify as a habitat. The second part expresses the spatial needs of the species. Within the fixed neighboring area of a stand, a certain percentage of the area (corresponding to a species' required amount of habitat and spatial distribution) should fulfill the specified stand conditions. The neighboring area is defined as the area within a circle with a radius of a specified number of metres from the centroid of the stand (Fig. 1).

The habitat model is included in a traditional forest planning problem consisting of selecting management activities (e.g. thinning, clear-felling or do nothing) for every stand in the landscape during a planning horizon so that the net present value (NPV) from future activities is maximized. This objective is subject to that only a certain percentage of the area is to be felled each period and to the demand for a certain habitat area. As can be seen in the formulation below, the problem formulation is built on the concept of a treatment schedule. Consequently, the model is an example of a standard model I formulation (Johnson and Scheurman, 1977), except for the habitat demands.

The mathematical formulation of the problem is as follows: 
$\operatorname{Max} Z_{1}=\sum_{i \in I} \sum_{j \in J_{i}} D_{i j} X_{i j}$

$$
\begin{aligned}
& h_{i p} \leq \sum_{j \in M_{i p}} x_{i j} \\
& \forall i \in I, \forall p \in P \\
& T h_{i p} \leq \sum_{l \in L_{i}} \sum_{j \in M_{l p}} a_{i l} x_{i j} \\
& \forall i \in I, \forall p \in P \\
& \sum_{i \in I} A_{i} h_{i p} \geq \bar{H}_{p} \\
& \forall p \in P \\
& h_{i p}=\{0,1\} \\
& \forall i \in I, \forall p \in P \\
& \sum_{i \in I} \sum_{j \in N_{i p}} A_{i} X_{i j} \leq \bar{R}_{p} \\
& \forall p \in P \\
& \sum_{j \in J_{i}} x_{i j}=1 \\
& \forall i \in I \\
& x_{i j}=\{0,1\} \\
& \forall i \in I, \forall j \in J_{i}
\end{aligned}
$$




$$
\begin{array}{ll}
P & =\text { set of periods in the planning horizon } \\
N_{i p} & =\text { set of treatment schedules for stand } i \text { that cause clear cutting in } \\
& \text { period } p \\
D_{i j} & =\text { NPV from period } 1 \text { to infinity from stand } i \text { and treatment schedule } \\
T & = \\
& =\text { the minimum number of hectares within the neighboring area of a } \\
& \text { stand that should fulfill the stand-wise habitat conditions before the } \\
& =\text { the amount of area for stand } l \text { that is included in the neighboring } \\
a_{i l} & =\text { area of stand } i \\
& =\text { area for stand } i \\
\bar{R}_{p} & =\text { minimum number of demanded hectare of habitat in period } p \\
\bar{H}_{p} &
\end{array}
$$

Equation 1 specifies the objective function, i.e. to maximize the NPV from future management activities. Equations 2-5 are connected to the habitat demands. To describe if a stand consists of habitat in a certain period, an indicator variable, $h_{i p}$, is used. When this variable, $h_{i p}$, takes the value 1 , stand $i$ consists of habitat in period $p$. Equation 2 specifies that the variable $h_{i p}$ could only take the value of 1 if stand $i$ is managed with treatment schedules that cause stand-wise habitat conditions in period $p$. Equation 3 specifies that $h_{i p}$ could only take the value of 1 if a minimum number of hectares, $\mathrm{T}$, within the neighboring area of stand $i$ are managed with treatment schedules that fulfill the stand-wise habitat conditions. It should be noted that both equations 2 and 3 must be fulfilled before stand $i$ qualifies as a habitat and the variable $h_{i p}$ could take the value of 1 . If the variable 
$h_{i p}$ could take the value of 1 , in line with equation 2 and 3 , equation 4 will force $h_{i p}$ to take the value of 1 , as long as the demand for a minimum number of hectares with habitat (equation 4) is not fulfilled. Equation 5 specifies that the variable $h_{i p}$ could only take the value of 0 or 1 . Equation 6 ensures that the clearcut area in each period not exceed a certain number of hectare. Finally, equations 7 and 8 ensure that all stands are assigned exactly one treatment schedule.

\section{Case study}

To illustrate this approach, we applied the general habitat model to the habitat needs for Hazel Grouse in the Krycklan watershed (6414’’ N, 1946’ E) approximately $50 \mathrm{~km}$ northwest of Umeå, northern Sweden . This area was selected because it is, in many respects, typical for managed forest in this part of Sweden and it represents a size relevant for management of larger forest holdings. Furthermore, we had access to detailed forest data for this area. The total area is 6700 hectares of which 6098 hectares is productive forestland divided into 904 stands. The forest data for the area was estimated by the $k$-nearest neighbor method $(k N N)$ (Reese et al., 2003) with stands delineated with the algorithm developed by Hagner (1990). The major part of the Krycklan watershed is forested with Norway spruce (Picea abies L.) in low-lying areas and Scots pine (Pinus sylvestris L.) in dryer upland areas.

\section{Habitat model for Hazel Grouse}

Hazel Grouse is a game species that has been proposed as an indicator of forest biodiversity at the landscape level (Jansson and Andrén, 2003). It is used as indicator of landscape continuity in the European NATURA 2000 network and also as indicator of high natural values of forests in Sweden's work on environmental quality objectives. The link between forest and landscape 
management and the species occurrence has been quantified and modeled in several countries (Åberg et al., 2003; Mörtberg and Karlström, 2005; Mathys et al., 2006; Müller et al., 2009; Longru et al., 2010). In Fennoscandia, Hazel Grouse inhabits mixed forests and mostly feed on buds and catkins of deciduous trees, viz. downy Birch (Betula pubescens) and alder (Alnus glutinosa and A. incana), and use norway Spruce (Picea abies) for cover (Åberg et al., 2003). The foraging resources and cover must be in close proximity to fulfill the habitat needs of Hazel Grouse (Swenson, 1993). One pair requires 15-25 ha of suitable habitat with interpatch distance usually limited to a few hundred meters ( $₫$ berg et al., 1995). Therefore, in landscapes that lack large habitat patches (e.g. managed forests), the distance to and degree of resource dispersion in neighboring forest stands thus define, along with stand quality, habitat suitability for Hazel Grouse. We defined Hazel Grouse habitat as forest stands containing at least 25\% spruce and $10-40 \%$ deciduous trees (by volume) that were at least 20 years old. To accommodate the spatial aspect (i.e. landscape level requirement), we followed thr recommendation for the species made by Angelstam et al., 2004 that there should be at least 20 ha of suitable habitat within $565 \mathrm{~m}$ (100 ha) from the center of each stand (i.e. landscape threshold set at 20\%, T=20). This approach des not explicitly employ inter-patch distance as a parameter; however, we assume that used landscape level threshold and species-specific scale employed (100 ha) account for the spatial dispersion of habitat and its connectivity. In addition, the study area is a contiguous forest land and in this kind of matrix, Hazel Grouse is moving more efficiently between habitat patches in comparison with semi-open landscapes (Åberg et al., 1995). Stands that did not fulfill these requirements were considered unsuitable (i.e. stands were classified as either habitat or nonhabitat). 


\section{Generation of treatment schedules}

The planning horizon was set to 100 years and divided into 20 periods of 5 years (i.e. $P$ was set to 20). Treatment schedules and future forest conditions (e.g. age and volume of spruce and birch, etc.) for the stands were simulated using the Heureka system. The Heureka system is a newly developed planning system for multiple-use forestry (Heureka, 2008). Each treatment schedule consists of different timing for the allowed silviculture activities (i.e. thinning and clearcutting with appropriate regeneration following a harvest). For each stand, schedules that favored timber production, schedules that favored the stand-wise conditions of Hazel Grouse and a no-treatment alternative were generated. In total, 22167 treatment schedules were generated, or an average of 25 schedules for each stand. Economic data used (timber, regeneration, and harvesting costs) for calculating the NPV for each schedule were based on a timber price list retrieved from the 2008 pricelist for the forest owners organization in northern Sweden and were considered representative for the region. NPVs were based on a $3 \%$ discount rate.

\section{Optimization algorithm}

The stated problem was solved using a traditional branch and bound algorithm. The model was formulated with the AIMMS 3.8 optimization modeling system (Bisschop and Roelofs, 1999), and solved from within AIMMS with ILOG ${ }^{\mathrm{TM}}$ CPLEX 11.0. The software was run on a PC with a $2.8 \mathrm{GHz}$ Pentium 4 processor and $1.5 \mathrm{~GB}$ of RAM. In the case study, a convergence bound of $1 \%$ was used. 


\section{Results}

Besides the basic definition for Hazel Grouse, where $\mathrm{T}$ is set to 20 hectares, the stated management problem was solved for two additional values on T; 0 and 40 hectares (Table 1). This was done to investigate the possibilities of creating different spatial layouts with different degrees of clustering of the habitat. A T value on 0 hectares, represents a situation where no spatial consideration is included in the definition for habitat, and a $\mathrm{T}$ value on 40 hectares, represents a situation with quite high spatial consideration. With a $\mathrm{T}$ value on 0 the initial amount of habitat is 1374 ha, a T value on 20 gives 1118 ha and a $\mathrm{T}$ value on 40 gives 559 ha. Irrespective of the value of $\mathrm{T}$, the minimum habitat demand was set to be $10 \%$ of the productive area in the end of all periods (i.e. $\bar{H}_{p}$ was set to 610 for all periods in equation 4). In all three cases the accepted clear-cut area in each period was set to $10 \%$ of the productive landscape area (i.e. $\bar{R}_{p}$ in equation 6 was set to 610 for all periods).

The amount of habitat is, because of the restriction, always equal to or more than 610 ha. For cases $b$ and $c$ the amount of habitat is balancing on the demand in almost every period. However, for case a, the amount of habitat is decreasing over time (Fig. 2). In order to analyze the resulting layout of habitat over time, the number of habitat areas and the mean and maximum size of these areas were calculated. A habitat area is here defined as a group of contiguous stands where the stands fulfill the stand-wise conditions and the spatial definitions in line with the definition of $\mathrm{T}$ for being a habitat. The value of $\mathrm{T}$ had a clear effect on the resulting spatial layout of the habitat. This is reflected in the number of areas as well as in the mean, maximum, and minimum size of habitat areas after harvest in 
a planning period (Fig. 3). The differences in the spatial layout could also been clearly seen in the habitat maps for period 10 (Fig. 4). When no spatial consideration is included (case a), the habitats are spread out all over the area with many small habitats. With an increased value of $\mathrm{T}$, stands consisting of habitat become more aggregated and the number of small isolated habitats decreases.

To evaluate whether the proposed model had any effect on the harvest levels, the predicted harvest volumes in each period for each case were recorded (Fig. 5). It seems that the effect both on total harvest volumes and over time with an increased T value is modest. The amount of clear- cut area is, because of the restriction, equal to or less than 610 hectare in every period. The trends over time for clearcut area follow the trend for harvest volume for all three cases.

The reduction in NPV with an increased T value is shown in Table 1. It appears that it is possible to create aggregated habitats with a moderate decrease in NPV. Finally, the solution times are shown in Table 1. The solution times increase with an increased $\mathrm{T}$ value.

\section{Discussion}

This study illustrates one modeling approach for including spatial aspects of habitat, in addition to stand-wise factors, in an optimization model that could be solved with exact optimization methods. To be able to use exact solution methods could be an advantage even if an exact solution method does not necessarily produce the optimal solution to the real forest management planning problem but only the exact solution to the formulated model of the planning problem. 
The alternative to using exact methods could be to using heuristic methods. However, there are problems with using a heuristic method, e.g. when dealing with many constraints because of problems in the relative weighting of these functions. Further, since the resulting problem could easily be solved with e.g., a traditional branch and bound algorithm, it could be included in available forest planning systems building on exact solution methods such as the Heureka system (Heureka, 2008). Forest managers could then produce different long-term plans with traditional timber production objectives and habitat demands for different species. As a result, it could work as a tool to learn more about the trade-off between different objectives such as NPV and available habitat area. We consider this an important and necessary step to better manage biodiversity in forest planning.

Habitat suitability models are, by necessity, simplifications of reality, focusing only on a limited number of factors deemed important for species to meet their life requirements. For example, habitat suitability models do not take biotioc interactions into consideration (Van Horne, 2002). Despite these shortcomings, the strength of a generalized habitat model, like the one presented in this paper, is that it could easily be adapted to the needs of other species by changing the definitions of the stand-wise and spatial conditions. An extension of this would be to develop models for generic habitat suitability indicators, i.e. "synthetic species" that incorporates the needs of many real world species (Hirzel et al., 2001). Further, by including habitat demands for different species, the model presents the possibilities of optimizing for the needs of multiple species. However, in such cases, the number of variables and constraints will increase. This could also lead to that there will be conflicting demands between the different species that 
increase the complexity of the problem and the problem will be more difficult to solve. Another strength of the model is that it could be combined with other restrictions used in traditional forest planning and the problem could still be solved with exact solution methods. However, including, e.g. even wood flow constraints would probably increase the solution time. In a case study by Öhman and Eriksson 2010, it was indicated that when the wood flow restrictions were excluded from the problem, the solution time decreased substantially.

The results of the case study showed that it is possible to increase the clustering of habitats by increasing the $\mathrm{T}$ value (i.e. the number of hectares within the neighboring area of a stand that fulfill the stand-wise habitat conditions before the stand could qualify as a habitat). Maintaining a clustered arrangement of habitats in a fragmented landscape is essential for many species, since it is linked to levels of connectivity (e.g. D'Eon et al., 2002) and considered a positive step for biodiversity management (Moilanen and Wintle, 2006). Further, the effect on harvest volume and the cost of increasing the clustering seems to be limited. In our study, the harvest pattern was almost the same in all three cases. The number of habitat patches, on average over the periods, was 91 for case a, 29 for case b and 12 for case c. Despite this spatial consideration, the reduction in NPV between cases $\mathrm{a}$ and $\mathrm{b}$ was negligible and the reduction in NPV between case a and c was only $3.9 \%$. This is consistent with other studies showing that the cost for including spatial consideration seems to be small (Nelson and Finn, 1991; Yoshimoto and Brodie, 1994; Öhman and Wikström, 2008). One explanation for the relatively low cost in the case study is the definition of habitat connected to the stand-wise conditions that results in a relatively good supply of potential habitat for Hazel Grouse over time in the landscape. With more demanding stand- 
wise definitions, e.g. an increased minimum limit for the proportion of deciduous trees or an increased age definition the amount of stands that could qualify as habitats would decrease and the cost would increase.

Even if the results from the case study seem to be promising, they raise a few issues that need to be discussed. First, all possible treatment schedules were not generated in the case study even if the schedules that were generated were the most practicable. It had been possible to generate more schedules since there is nothing in the model formulation that prevents this. However, this could lead to that the solution time increases since the problem size increases. More available schedules would also probably lead to that the reduction in NPV would have decrease if these additional schedules favour both timber production and habitat production. Second, the projected habitat areas do not necessarily "connect" over time. However, the habitats in one period are, in most cases, adjacent to or overlap with the habitats of the following period even if there is no guarantee this will occur. Third, the case study that we used applied the model only to Hazel Grouse. However, we argue that Hazel Grouse is a well-studied species of high societal interest being also a good indicator of forest value from a biodiversity perspective and already used as a tool in practical management of biodiversity (Jansson and Andrén, 2003; Longru et al., 2010). In addition, as pointed out the habitat parameters in the model could be changed so as to accommodate the needs of other species. Next, the definition of suitable habitat for Hazel Grouse used in this study applied cutoff values, and a higher degree of realism would have been achieved if we had used continuous data. Better realism could also have been achieved if we had access to more detailed data on tree species composition; alder, for example, is an important food substrate for Hazel Grouse, but it was not 
possible to distinguish this species in the forest database. The quality of habitat data in terms of spatial and thematic resolution is still not satisfying. Manton et al. (2005) demonstrated large discrepancies among different forest data based on the remote sensing having large consequences for the habitat suitability modeling. Hopefully, new technologies used for describing forest structure and composition (e.g. airborne laser scanning) will allow for better match between ecological requirements of species and available habitat data in habitat suitability modeling in the future (e.g. Breidenbach et al., 2010).

Since fragmentation and loss of habitat are two of the largest threats to biological diversity in forested landscapes (Fahrig, 2003), establishing physical connectivity of habitats is a necessary step in landscapes with limited amounts of suitable habitat remaining (Mikusiński et al., 2007). The habitat suitability models for species with larger area requirements are important tools for achieving this. We hope that the concept presented here for including consideration of habitat demands, where the habitat model includes both stand-wise and spatial definitions, will contribute to the available tools for including ecological requirements of species in forest management planning.

\section{Acknowledgements}

This paper emanates from the Biodiversity Assessment Modeling project and the optimization project within the Heureka Research Program, funded by the Foundation for Strategic Environmental Research (MISTRA) and Swedish University of Agricultural Sciences. 


\section{References}

Åberg, J., Jansson, G., Swenson, J.E., and Angelstam, P. 1995. The effect of matrix on the occurrence of hazel grouse (Bonasa bonasia) in isolated habitat fragments. Oecologia (Berl.), 103(3): 265.269. doi: 10.1007/BF00328613.

Åberg, J., Swenson, J.E., and Angelstam, P. 2003. The habitat requirements of hazel grouse (Bonasa bonasia) in managed boreal forest and applicability of forest stand descriptions as a tool to identify suitable patches. For. Ecol. Manage. 175(1-.3):

437.444. doi: 10.1016/S0378-1127(02)00144-5.

Angelstam, P., Roberge, J.-M., Lohmus, A., Bergmanis, M., Brazaitis, G., Donz-Breuss, M., Edenius, L., Kosinski, Z., Kurlavicius, P., Larmanis, V., Lukins, M., Mikusinski, G., Ra.inski, E., Strazds, M., and Tryjanowski, P. 2004. Habitat modelling as a tool for landscape-scale conservation- . a review of parameters for focal forest birds.

Ecological. Bulletins., 51: 427.453.

Atamturk, A., and Savelsbergh, M.W.P. 2005. Integer programming software systems. Ann. Oper. Res. 140(1): 67.124. doi: 10.1007/s10479-005-3968-2.

Bettinger, P., Johnson, D.L., and Johnson, K.N. 2003. Spatial forest plan development with ecological and economic goals. Ecol. Model. 169(2-.3): 215.236. doi: 10.1016/ S0304-3800(03)00271-0.

Bisschop, J., and Roelofs, M. 1999. AIMMS Uuser fs Gguide. Paragon Decision Technology, B.V., Haarlem, The Netherlands.

Breidenbach, J., Nasset, E., Lien, V., Gobakken, T., and Solberg, S. 2010. Prediction of species specific forest inventory attributes using a nonparametric semi-individual tree crown approach based on fused airborne laser scanning and multispectral data. Remote Sens. Environ. 114(4): 911.924. doi: 10.1016/j.rse.2009.12.004.

D' Eon, R.G., Glenn, S.M., Parfitt, I., and Fortin, M.-J. 2002. Landscape connectivity as a function of scale and organism vagility in a real forested landscape. Conserv. Ecol. 6(2): 10 [online]. Available from http://www.consecol.org/vol6/iss2/art10/. 
Edenius, L., and Mikusi.ski, G. 2006. Utility of habitat suitability models as biodiversity assessment tools in forest management. Scand. J. For. Res. 21(Suppl. 7): 62.72. doi: 10.1080/14004080500486989.

Fahrig, L. 2003. Effects of habitat fragmentation on biodiversity. Annu. Rev. Ecol. Syst. 34(1): 487.515. doi: 10.1146/annurev.ecolsys.34.011802.132419.

Felix, A.B., Campa, H., III, Millenbah, K.F., Winterstein, S.R., and Moritz, W.E. 2004.

Development of landscape-scale habitat-potential models for forest wildlife planning and

management. Wildl. Soc. Bull. 32(3): 795.806. doi: 10.2193/0091-7648(2004)032[0795: DOLHMF]2.0.CO;2.

Fries, C., Carlsson, M., Dahlin, B., Lamas, T., and Sallnas, O. 1998. A review of conceptual landscape planning models for multiobjective forestry in Sweden. Can. J. For. Res. 28(2): 159.167. doi: 10.1139/cjfr-28-2-159.

Gustafson, E.J., Roberts, L.J., and Leefers, L.A. 2006. Linking linear programming and spatial simulation models to predict landscape effects of forest management alternatives.

J. Environ. Manage. 81(4): 339.350. doi: 10.1016/j.jenvman.2005.11.009.

Hagner, O. 1990. Computer aided stand delineation and inventory based on satellite remote sensing. In The usability of remote sensing for forest inventory and planning. Edited by R. Sylvander, and S. Poso. Report. No. 4. Remote Sensing Laboratory, Swedish University of Agricultural Sciences, Umeå, Sweden. Heureka. 2008. The Heureka Research Program [online]. Stockholm, Sweden: Foundation for Strategic Environmental Research (MISTRA);, Stockholm, Sweden. c2009. Available from http://www.mistra.org/heureka [accessed 9 October 2008 Oct 09]. Heywood, W.H. (Editor). 1995. Global biodiversity assessment. Cambridge University Press, Cambridge, U.K. Hirzel, A.H., Helfer, V., and Metral, F. 2001. Assessing habitat-suitability models with a virtual species. Ecol. Model. 145(2-.3): 111.121. doi: 10.1016/S0304-3800(01)00396-9. 
Hof, J., Bevers, M., Joyce, L., and Kent, B. 1994. An integer programming approach for spatially and temporally optimizing wildlife populations. For. Sci. 40: 177.191.

Hurme, E., Kurttila, M., Monkkonen, M., Heinonen, T., and Pukkala, T. 2007.

Maintenance of flying squirrel habitat and timber harvest: a site-specific spatial model in forest planning calculations. Landsc. Ecol. 22(2): 243.256. doi: 10.1007/s10980-0069019-9.

Jansson, G., and Andren, H. 2003. Habitat composition and bird diversity in managed boreal forests. Scand. J. For. Res. 18(3): 225.236. doi: 10.1080/02827580308622. Johnson, E.L., Nemhauser, G.L., and Savelsbergh, M.W.P. 2000. Progress in linear programming-based algorithms for integer programming: an exposition. INFORMS J. Comput. 12(1): 2.23. doi: 10.1287/ijoc.12.1.2.11900.

Johnson, K.N., and Scheurman, H.L. 1977. Techniques for prescribing optimal timber harvest and investment under different objectives: discussion and synthesis. For. Sci. Mon. 18: 1.31.

Larson, M.A., Thompson, F.R., III, Millspaugh, J.J., Dijak, W.D., and Shifley, S.R. 2004. Linking population viability, habitat suitability, and landscape simulation models for conservation planning. Ecol. Model. 180(1): 103.118. doi: 10.1016/j. ecolmodel.2003.12.054.

Larsson, S., and Danell, K. 2001. Science and the management of boreal forest biodiversity. Scand. J. For. Res. 16(Suppl. 3): 5.9. doi: 10.1080/028275801300090528. Longru, J., He, H.S., Yufei, Z., Rencang, B., and Keping, S. 2010. Assessing the effects of management alternatives on habitat suitability in a forested landscape of northeastern

China. Environ. Manage. 45(5): 1191.1200. doi: 10.1007/s00267-010-9473-7. Manton, M.G., Angelstam, P., and Mikusi.ski, G. 2005. Modelling habitat suitability for deciduous forest focal species - . Aa sensitivity analysis using different satellite land cover data. Landsc. Ecol. 20(7): 827.839. doi: 10.1007/s10980-005-3703-z. Marzluff, J.M., Millspaugh, J.J., Ceder, K.R., Oliver, C.D., Withey, J., McCarter, J.B., 
Mason, C.L., and Comnick, J. 2002. Modeling changes in wildlife habitat and timber revenues in response to forest management. For. Sci. 48: 191.202.

Mathys, L., Zimmermann, N.E., Zbinden, N., and Suter, W. 2006. Identifying habitat suitability for hazel grouse Bonasa bonasia at the landscape scale. Wildl. Biol. 12(4): 357.366. doi: 10.2981/0909-6396(2006)12[357:IHSFHG]2.0.CO;2.

Mikusi.ski, G., and Edenius, L. 2006. Assessment of spatial functionality of old forest in Sweden as habitat for virtual species. Scand. J. For. Res. 21(Suppl. 7): 73.83. doi: 10.1080/14004080500487045.

Mikusiski, G., Pressey, R.L., Edenius, L., Kujala, H., Moilanen, A., Niemela, J., and Ranius, T. 2007. Conservation planning in forest landscapes of Fennoscandia and an approach to the challenge of Countdown 2010. Conserv. Biol. 21(6): 1445.1454. doi: 10.1111/j.1523-1739.2007.00833.x.

Moilanen, A., and Wintle, B.A. 2006. Uncertainty analysis favours selection of spatially aggregated reserve networks. Biol. Conserv. 129(3): 427.434. doi: 10.1016/j. biocon.2005.11.006.

Mortberg, U., and Karlstrom, A. 2005. Predicting forest grouse distribution taking account of spatial autocorrelation. J. Nat. Conserv. 13(2-.3): 147.159. doi: 10.1016/j. jnc.2005.02.008.

Muller, D., Schroder, B., and Muller, J. 2009. Modelling habitat selection of the cryptic Hazel Grouse Bonasa bonasia in a montane forest. J. Ornithol. 150(4): 717.732. doi: 10.1007/s10336-009-0390-6.

Nelson, J.D., and Finn, S.T. 1991. The influence on cut-block size and adjacency rules on harvest levels and road network. Can. J. For. Res. 21(5): 595.600. doi: 10.1139/x91081.

O'Connor, R.J. 2002. The conceptual basis of species distribution modeling: time for a paradigm shift? In Predicting Sspecies Ooccurrence: issues of accuracy and scale. Edited by J.M. Scott, P.J. Heglund, M.L. Morrison, J.B. Haufler, M.G. Raphael, W.A. Wall, and F.B. Samson. Island Press, Washington, D.C. pp. 25.33. 
Ohman, K., and Eriksson, L. 2010. Aggregating harvest activities in long term forest planning by minimizing harvest area perimeters. Silva Fenn. 44(1): 77.89.

Ohman, K., and Wikstrom, P. 2008. Incorporating aspects of habitat fragmentation into long-term forest planning using mixed integer programming. For. Ecol. Manage. 255(3-.4): 440.446. doi: 10.1016/j.foreco.2007.09.033.

Rebain, S., and McDill, M.E. 2003. A mixed-integer formulation of the minimum patch size problem. For. Sci. 49: 608.618.

Reese, H., Nilsson, M., Pahen, T.G., Hagner, O., Joyce, S., Tingelof, U., Egberth, M., and Olsson, H. 2003. Countrywide estimates of forest variables using satellite data and field data from the national forest inventory. Ambio, 32: 542.548.

Spies, T.A., McComb, B.C., Kennedy, R.S.H., McGrath, M.T., Olsen, K., and Pabst, R. J. 2007. Potential effects of forest policies on terrestrial biodiversity in a multiownership province. Ecol. Appl. 17(1): 48.65. doi: 10.1890/1051-0761(2007)017[0048:

PEOFPO]2.0.CO;2.

Swenson, J.E. 1993. The importance of alder to hazel grouse in Fennoscandian boreal forest: evidence from four levels of scale. Ecography, 16(1): 37.46. doi: 10.1111/j.16000587.1993.tb00057.x.

Tikkanen, O.P., Heinonen, T., Kouki, J., and Matero, J. 2007. Habitat suitability models of saproxylic red-listed boreal forest species in long-term matrix management: costeffective measures for multi-species conservation. Biol. Conserv. 140(3-.4): 359.372. doi: 10.1016/j.biocon.2007.08.020.

Tittler, R., Messier, C., and Burton, P.J. 2001. Hierarchical forest management planning and sustainable forest management in the boreal forest. For. Chron. 77: 998.1005. Turner, M.G., Gardner, R.H., and O’Neill, R.V. 2001. Landscape ecology in theory and practice. Springer, New York. Van Horne, B. 2002. Approaches to habitat modeling: the tensions between pattern and process and between specificity and generality. In Predicting species occurrence: 
issues of accuracy and scale. Edited by J.M. Scott, P.J. Heglund, M.L. Morrison, J.B. Haufler, M.G. Raphael, W.A. Wall, and F.B. Samson. Island Press, Washington, D.C. pp. 63.72 .

Vospernik, S., and Reimoser, S. 2008. Modelling changes in roe deer habitat in response to forest management. For. Ecol. Manage. 255(3-.4): 530.545. doi: 10.1016/j. foreco.2007.09.036.

Wei, Y., and Hoganson, H.M. 2007. Scheduling forest core area production using mixed integer programming. Can. J. For. Res. 37(10): 1924.1932. doi: 10.1139/X07-033.

Yoshimoto, A., and Brodie, J.D. 1994. Short- and long-term impacts of spatial restrictions on harvest scheduling with reference to riparian zone planning. Can. J. For. Res. 24(8): 1617.1628. doi: 10.1139/x94-210.

Zhang, H., Constantino, M., and Falcao, A. 2009. Modeling forest core area with integer programming. Ann. Oper. Res., Published online 15 January 2009. Available from http://www.springerlink.com/content/8382355m63hx841m/. doi: 10.1007/s10479-

009-0517-4. 
Table 1 Value for T, net present value (NPV), total harvest volume, and solution time for all investigated versions of the model formulation. $\mathrm{T}$ is the number of hectares within the neighboring area of a stand that should fulfill the standwise habitat conditions before the stand could qualify as a habitat. A T value of 0 represents a situation where no spatial consideration is included in the definition for habitat, a T value of 20 is the basic definition for Hazel Grouse, (Bonasa bonasia) used in the case study and a $\mathrm{T}$ value of 40 represents a situation with quite high spatial consideration

\begin{tabular}{rrrrr} 
Case & Value for T & NPV $\left(10^{6} \mathrm{SEK}^{2}\right)$ & Total harvest volume $\left(10^{3} \mathrm{~m}^{3}\right)$ & Solution time $(\mathrm{s})$ \\
\hline $\mathrm{a}$ & 0 & 118.9 & 1442 & 9.7 \\
$\mathrm{~b}$ & 20 & 118.5 & 1433 & 20.7 \\
$\mathrm{c}$ & 40 & 114.3 & 1364 & 188.4
\end{tabular}

${ }^{2}$ Net present value is in Swedish kronas (SEK) 
Figure captions

Fig. 1. The neighboring area is defined as the area within a circle with a radius of a specified numbers of metres from the center point of the stand.

Fig 2. Amount of habitat over the planning horizon.

Fig. 3. a) Number of habitat areas b) mean size of habitat areas, c) maximum size of habitat areas, and d) minimum size of the habitat areas after harvest in the end of each period during the planning horizon. It should be noted here that a habitat area is defined as a group of contiguous stands where the stands fulfill the standwise conditions and the spatial definitions for being a habitat.

Fig. 4. Spatial layout of the habitats areas after harvest at the end of period 10 for a) case a, b) case b, and c) case c.

Fig. 5. Harvest volumes over the planning horizon for cases a, b, and c. 
Fig. 1.

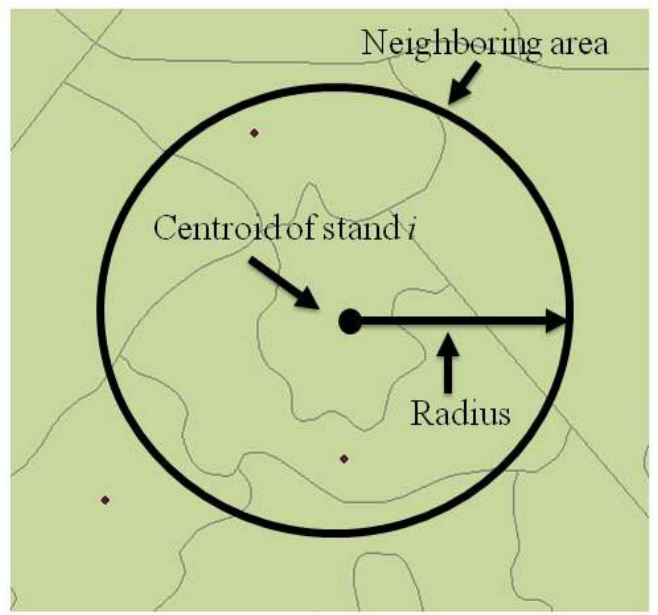

Fig. 2.

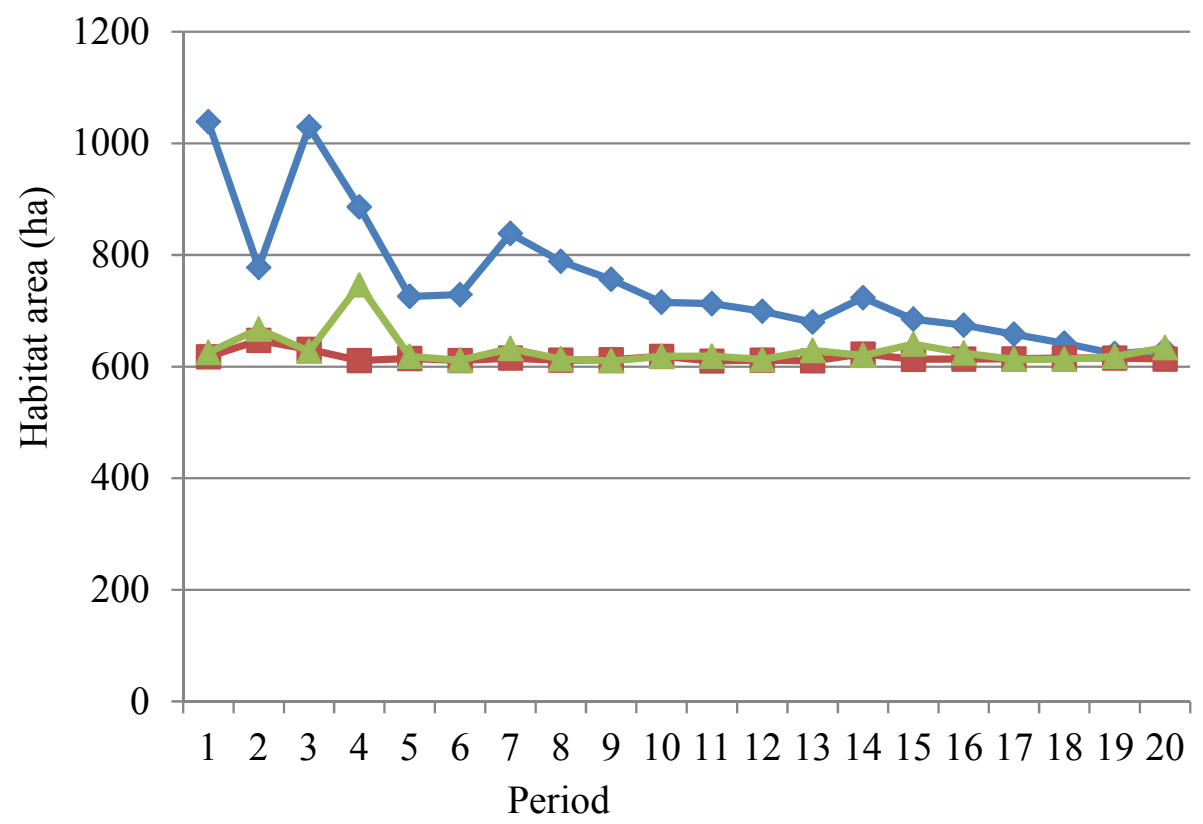

$\leadsto$ Case a - No spatial consideration

- -Case b - Medium spatial consideration

$\rightarrow$ Case c - High spatial consideraion 
Fig. 3.

a

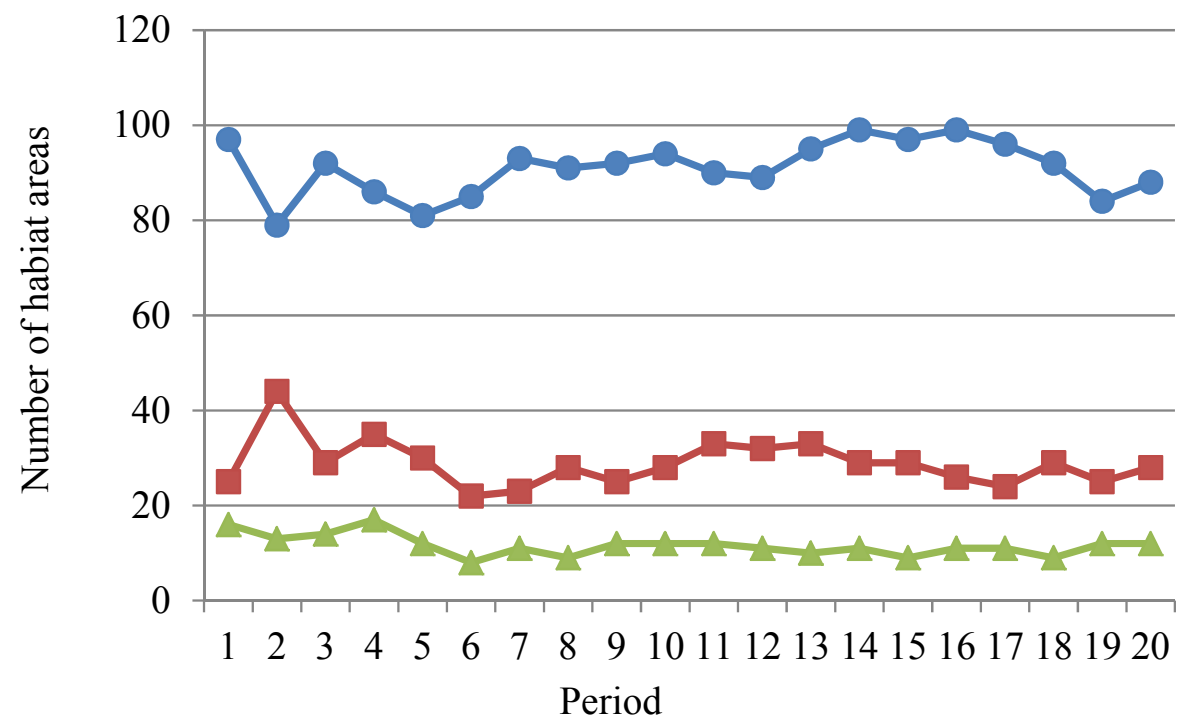

- Case a - No spatial consideration

- Case b - Medium spatial consideration

- Case c - High spatial consideraion

b

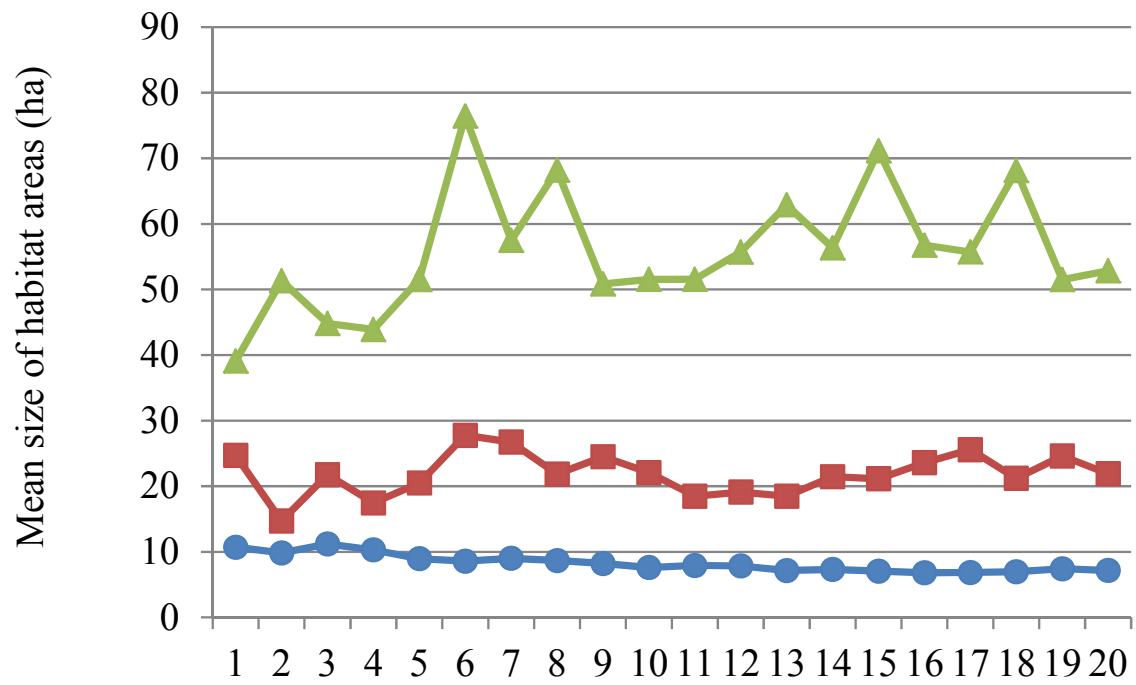

Period

-Case a - No spatial consideration

- Case b - Medium spatial consideration

$\rightarrow$ Case c - High spatial consideraion 


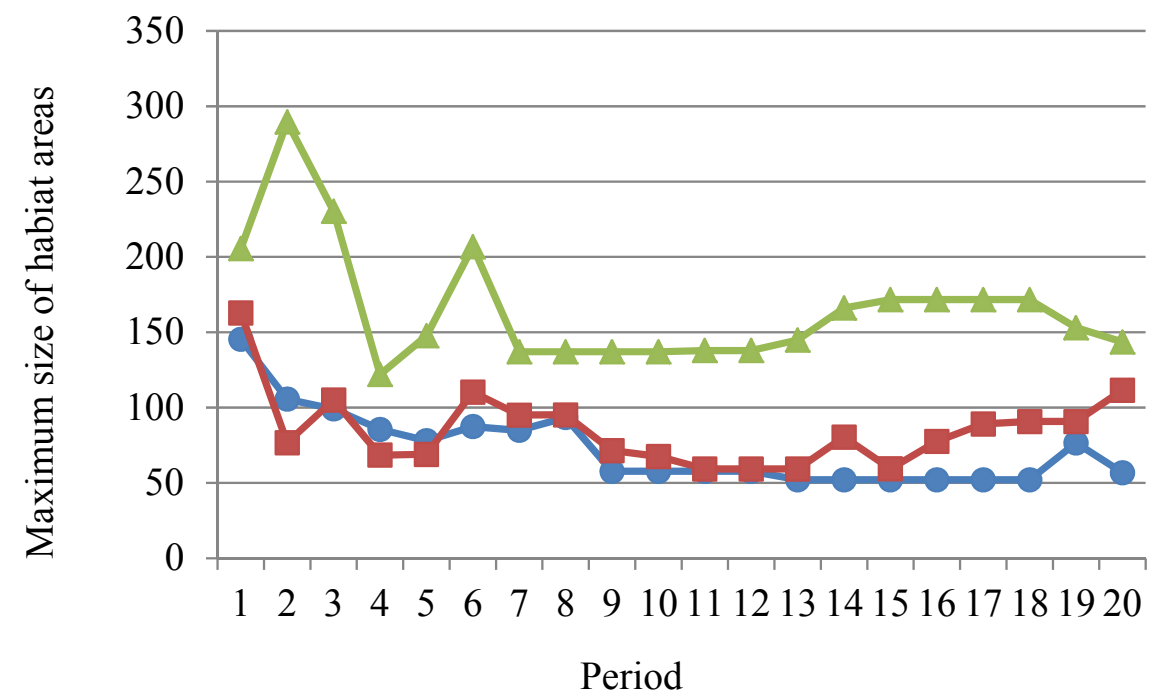

-Case a - No spatial consideration

- Case b - Medium spatial consideration

- Case c - High spatial consideraion

d

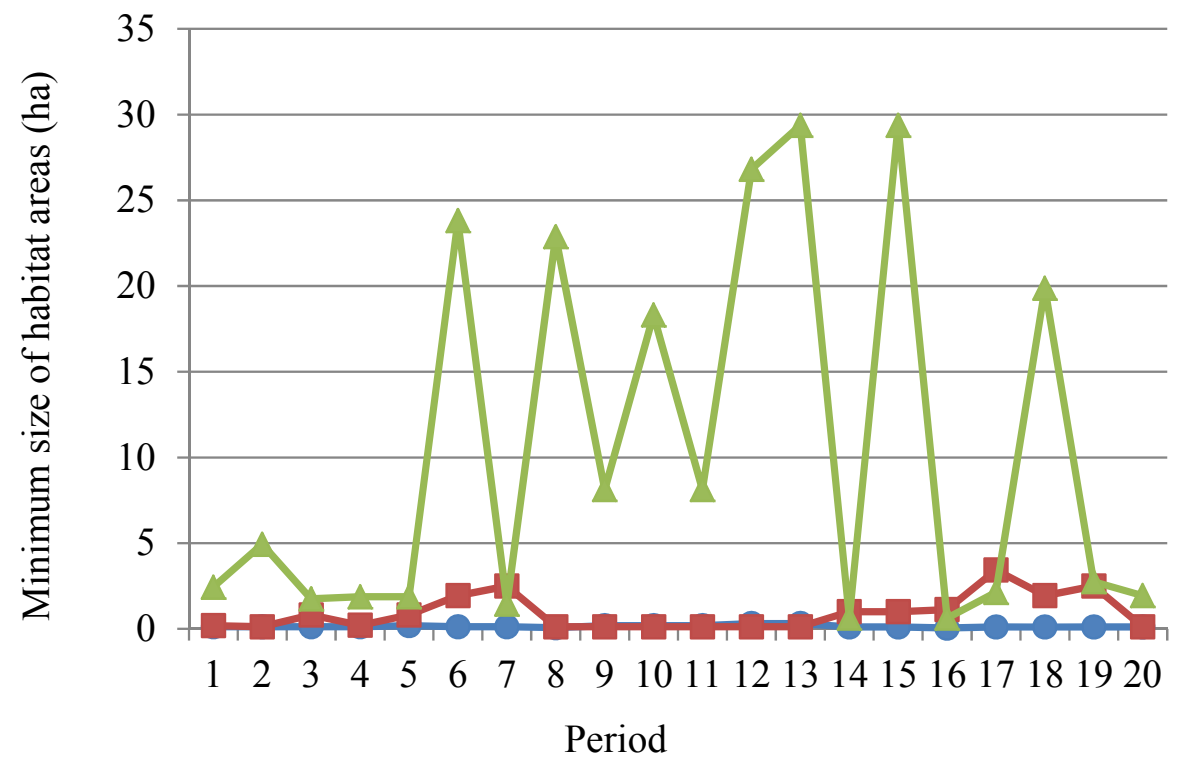

-Case a - No spatial consideration

- Case b - Medium spatial consideration

- Case c - High spatial consideraion 
Fig. 4.

a

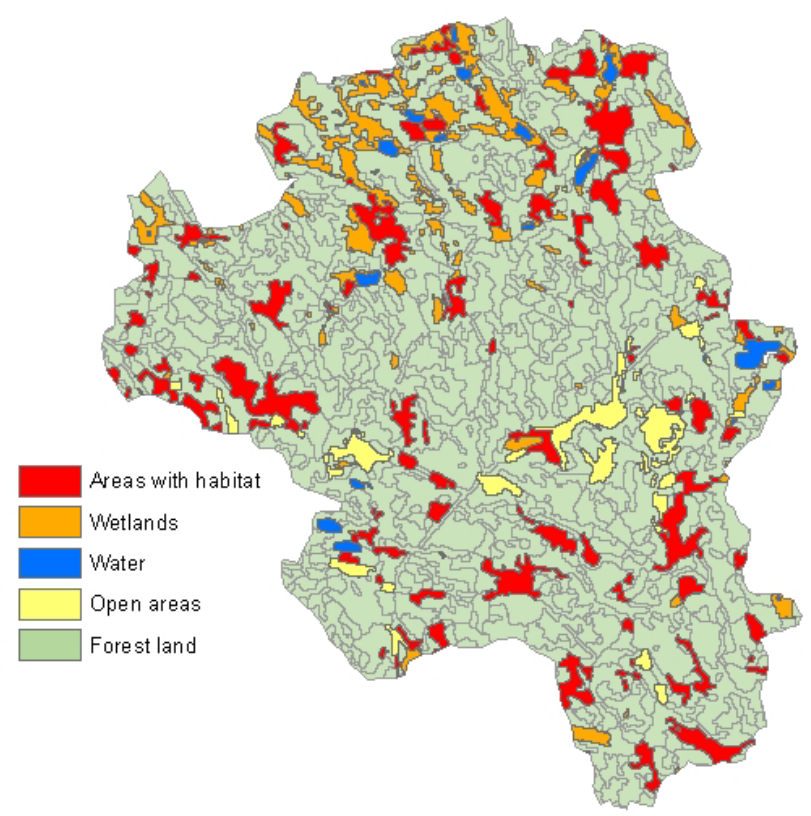

b

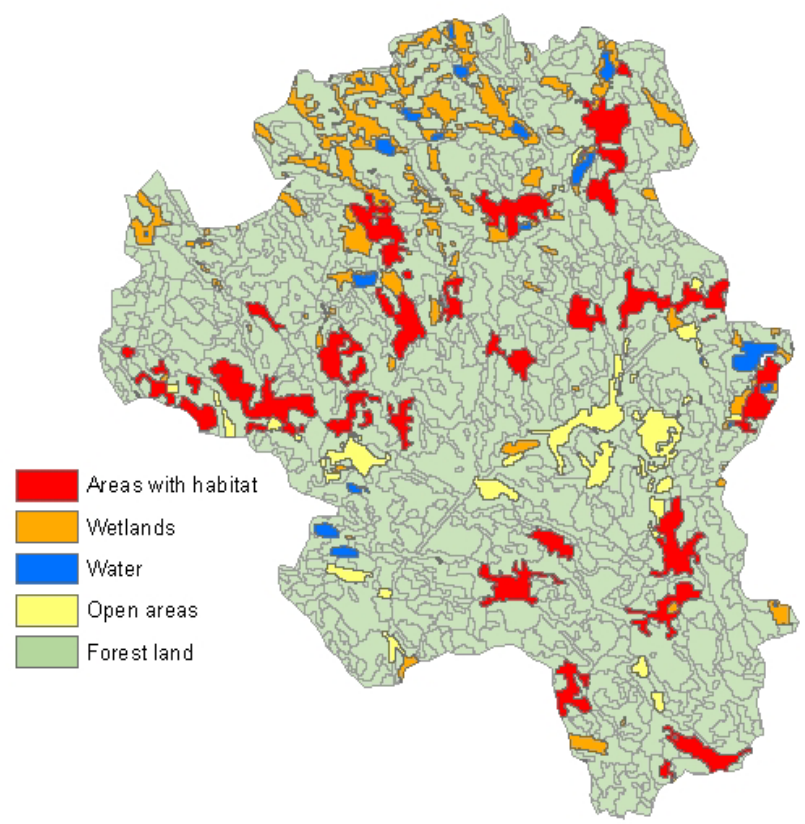




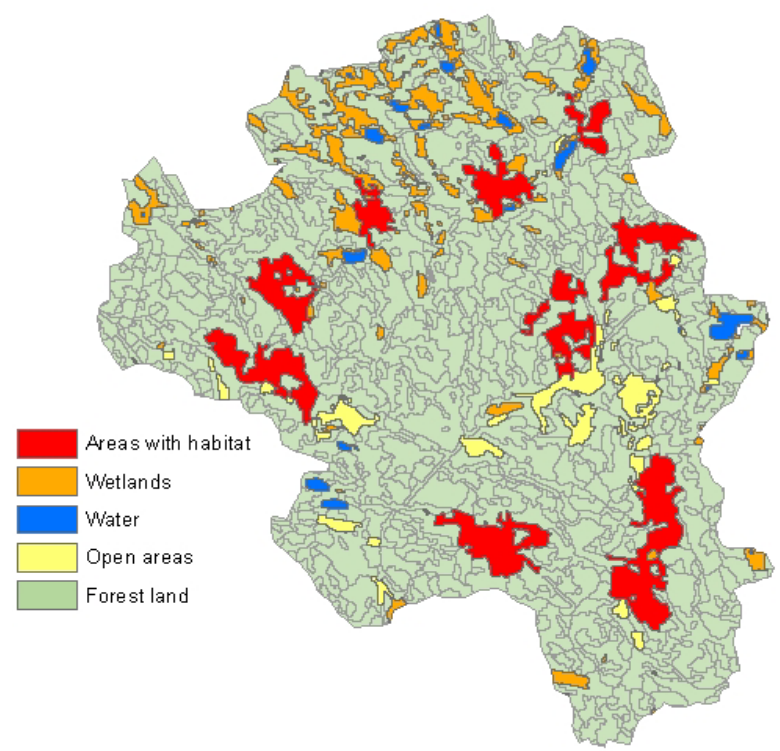


Fig. 5.

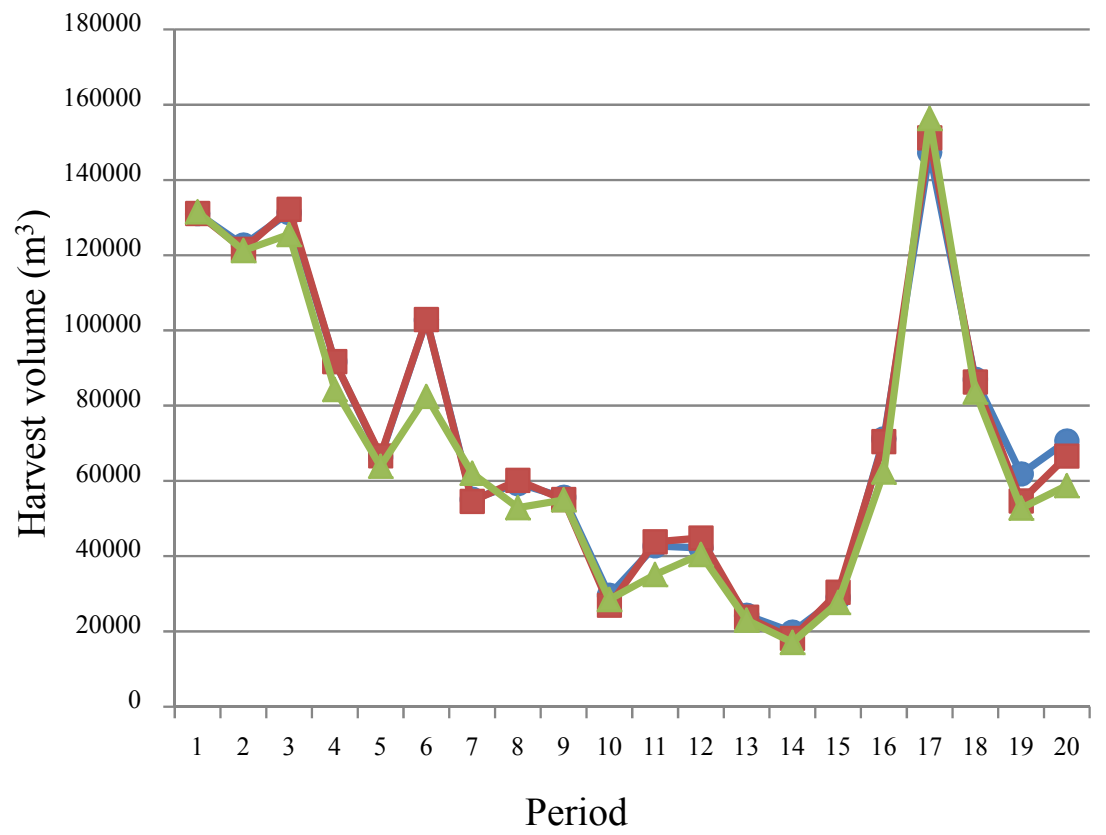

- Case a - No spatial consideration

- Case b - Medium spatial consideration

$\rightarrow$ Case c - High spatial consideration 